\title{
Optimization of Distributed Generation in Micro Grid using a Hybrid Metaheuristic Technique
}

\author{
K. Santhosh ${ }^{1}$, R. Neela ${ }^{2}$ \\ ${ }^{1}$ Department of Electrical Engineering, FEAT, Annamalai University, India, santhoshkasi86@gmail.com \\ ${ }^{2}$ Department of Electrical Engineering, FEAT, Annamalai University, India, neelaaueee@ gmail.com
}

\begin{abstract}
This paper introduces a hybrid metaheuristic approach with eagle strategy (ES) and particle swarm optimization (PSO) technique to minimize the operation cost of a low voltage micro grid with renewable energy source (RES) such as photovoltaic (PV), micro turbine (MT), fuel cell (FC), wind turbine (WT), and battery, while satisfying the load demand and system operating constraints. The cost optimization problem is developed as a nonlinearly constrained mathematical problem to optimize the generation of DGs in the micro grid. The presented hybrid approach is validated on a low voltage micro grid and its better execution property is compared with the other metaheuristic techniques for example PSO, genetic algorithm (GA), adaptive modified PSO (AMSPO) and chaotic PSO (CPSO).
\end{abstract}

Key words: Distributed generation, Eagle strategy, Micro grid, Particle swarm optimization, Renewable energy sources.

\section{INTRODUCTION}

Electricity is one of the most greatly utilized forms of energy globally. Power production comes from a variety of technologies, and the primary sources have a general law that the application of a rotary motion to produce electricity through generators.

The goal of electrical power producing system is to produce the only energy required to be consumed at given period, by taking into account of various voltage levels to be supplied and the power loss related with the distribution and transmission systems. Due to the globalization of this resource, power demand has grown greatly, making it necessary to raise community awareness about the environmental impacts caused by large power production industries with regard to more conventional or centralized generation. In view of this, it led to various developments in micro grids, where a particular interest is given to decentralized or distributed generation (DG) [1]. Even though this form of DG has several advantages, some factors need to be taken into account.
By adding DG to a micro grid, a set of different operating conditions is imposed on the system such as power balance constraints, power generation limits, voltage limits, failure levels, harmonic distortions and network stability problem that severely impact the operation of the entire power systems if proper care is not taken [2].

The potential advantages of DG production depend on the position and size of the DG unit. The proper rating and placing of DG will results in condition that reduced energy losses and increased reliability level of the complete network. Nevertheless, the determination of the optimal position and rating of the generation unit is a complex job and it is necessary to computationally study about the parameter by taking into account that the unpredictability and intermittence associated with renewable energies [3].

The study underlying this option must be undertaken to minimize the operating cost of DG or other objectives in the functions of DG placement while satisfying the system constraints [4]. With the growing introduction of DG in the electricity grid, it's optimized planning and management in the distribution system will make it possible to introduce smart grids, which is of great interest today in the world electrical sector [5].

In this paper, a hybrid metaheuristic approach with eagle strategy (ES) [6]-[9] and particle swarm optimization (PSO) [7] technique so called ES-PSO algorithm to minimize the operation cost of a low voltage micro grid with renewable energy source (RES) such as photovoltaic (PV), micro turbine (MT), fuel cell (FC), wind turbine (WT), and battery, while satisfying the load demand and system operating constraints [10], [11]. The cost optimization problem is developed as a nonlinearly constrained mathematical problem to optimize the generation of DGs in the micro grid.

The reminder of this paper is organized as follows. Section 2 depicts the problem formulation for the operating cost optimization problem in a low voltage micro grid. The proposed hybrid metaheuristic approach with ES and PSO are illustrated in section 3. The simulation results of proposed 
K. Santhosh et al., International Journal of Emerging Trends in Engineering Research, 8(9), September 2020, $5104-5110$

hybrid metaheuristic technique (ES-PSO) used to solve the cost optimization problem in micro grid is depicted in section 4. Finally, section 5 summarizes the conclusion and advantages of the proposed hybrid method in solving the micro grid optimization problems.

\section{COST OPTIMIZATION PROBLEM IN MICRO GRID}

The operating cost optimization problem in a low voltage micro grid is specified as a mathematical problem to schedule the optimum generation output power of DGs in micro grid and appropriate ON or OFF status of DGs to minimize the micro grid's total operation cost while meeting the power demand and other technical constraints. The numerical modeling of this problem is formulated in the following sections.

\subsection{Micro Grid Operating Cost}

The micro grid's total operating cost is consists of the generating cost of DG, start-down or shut-up cost of DGs and the cost of power interchanged between the main grid and micro grid, in Euro cent ( $€ \mathrm{ct}$ ). The objective of total operating cost minimization is directs at determining the optimal power flow from RESs to customer loads in a financial manner for 24 hour time horizon. The minimization of total operating cost of micro grid is derived as follows [3],

$$
\begin{aligned}
\operatorname{Min} f & =\sum_{t=1}^{T}\left\{\sum _ { i = 1 } ^ { N _ { g } } \left[u_{i}(t) P_{G i}(t) B_{G i}(t)+S_{G i} \mid u_{i}(t)\right.\right. \\
& \left.-u_{i}(t-1) \mid\right]+\sum_{j=1}^{N_{s}}\left[u_{j}(t) P_{s j}(t) B_{s j}(t)+S_{s j} \mid u_{j}(t)-u_{j}\right. \\
& \left.(t-1) \mid]+P_{\text {Grid }}(t) B_{G r i d}(t)\right\}
\end{aligned}
$$

where,

$P_{G}$ is the output real power of DGs;

$P_{S}$ is the charging or discharging power of batteries;

$P_{\text {Grid }}$ is the active power exchanged between the utility grid and micro grid;

$B_{S}$ and $B_{G}$ are the bidding cost of batteries and DGs respectively;

$B_{\text {Grid }}$ is the bidding cost of power exchanged between the utility grid and micro grid;

$S_{G}$ and $S_{S}$ denote the start-down or shut-up cost of batteries and DGs respectively;

The state variable $X$ consists of real power output of DGs and batteries, and its ON or OFF status. The state variable $X$ is expressed as [3],

$$
\begin{aligned}
& X=\left[P_{g}, U_{g}\right]_{1 \times 2 n T} \\
& P_{g}=\left[P_{G}, P_{S}\right] \\
& n=N_{g}+N_{s}+1
\end{aligned}
$$

where, $T$ denotes 24 hour time horizon, $P_{g}$ is the real power vector consists of output real power of all DGs and charge or discharge power of batteries; $N_{s}$ and $N_{g}$ are the number of battery units and DGs respectively; $\mathrm{n}$ is number of variables in state vector $X ; U_{g}$ is a status vector representing $\mathrm{ON}$ or $\mathrm{OFF}$ status of DGs for 24 hour time horizon. The vectors can be expressed as [3], [12],

$$
\begin{aligned}
& P_{G}=\left[P_{G 1}, P_{G 2}, \ldots, P_{G, N g}\right] \\
& P_{G}=\left[P_{G i}(1), P_{G i}(2), \ldots, P_{G i}(t), \ldots, P_{G i}(T)\right] ; i=1,2, \ldots, N_{g}+1 \\
& P_{s}=\left[P_{s 1}, P_{s 2}, \ldots P_{s, N s}\right] \\
& P_{s j}=\left[P_{s j}(1), P_{s j}(2), \ldots, P_{s j}(t), \ldots, P_{s j}(T)\right] ; j=1,2 \ldots, N_{s}
\end{aligned}
$$

where, $P_{s j}(t)$ and $P_{G i}(t)$ are the real power output of $j^{\text {th }}$ battery and $i^{\text {th }}$ DG unit at time $t$ respectively.

$U_{g}=\left[u_{1}, u_{2}, \ldots, u_{n}\right]=\left\{u_{i}\right\}_{1 \times n} \in\{0,1\} ;$

$u_{k}=\left[u_{k}(1), u_{k}(2), \ldots, u_{k}(t), \ldots, u_{k}(T)\right] ; k=1,2, \ldots, n$

where, $u_{k}(t)$ is ON or OFF state of $k^{\text {th }} \mathrm{DG}$ at time $t$.

\section{EAGLE STRATEGY WITH PARTICLE SWARM OPTIMIZATION ALGORITHM}

\subsection{Standard Particle Swarm Optimization Technique (PSO)}

PSO algorithm is a stochastic optimization technique created in 1995 to diagrammatically model the characteristics of a troop of birds [7].

All particles store its own coordinate points in the problem exploration space that are colligated with the best fitness solution that has been found so far and is called Particle best $_{t}, p_{i}$ $=\left[p_{i 1}, p_{i 2}, \ldots, p_{i n}\right]$.

The position of the particle is modified by including its updated velocity to its previous positioning and the updated velocity is expressed as,

$$
v_{i d}^{t+1}=\omega \cdot v_{i d}^{t}+\varphi_{1}\left(p_{g d}-x_{i d}^{t}\right)+\varphi_{2}\left(p_{i d}-x_{i d}^{t}\right)
$$

where, $v_{i}^{t+1}$ will be the new particle velocity; $v_{i}^{t}$ and $x_{i}^{t}$ is the previous velocity and position of the particle $i$, respectively.

In (5), $\varphi_{1}=c_{1} r_{1}$ and $\varphi_{2}=c_{2} r_{2}$, where $c_{1}$ and $c_{2}$ are two positive acceleration components called social and cognitive components, respectively; $\omega$ represents the inertia factor; $r_{I}$ and $r_{2}$ both are random coefficients uniformly distributed in on the interval $[0,1] ; d$ is the index of dimension; $t$ is iteration number. 
K. Santhosh et al., International Journal of Emerging Trends in Engineering Research, 8(9), September 2020, $5104-5110$

Then, the new particle position is expressed as,

$$
x_{i d}^{t+1}=x_{i d}^{t}+v_{i d}^{t+1}
$$

The PSO technique proves to be efficient in solving problems where the search space is continuous as a result of the position and velocity of the particle being updated [7].

\subsection{Pseudo Code of PSO Algorithm}

The pseudo code of PSO technique to solve minimization problem is given in Algorithm 1.

\section{Algorithm 1. PSO Pseudo code}

FOR each particle

Randomly initialize particle

END

DO WHILE

FOR each particle

Compute the objective value using (1).

IF the present objective value is lesser than the local best

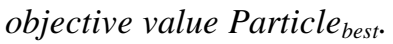

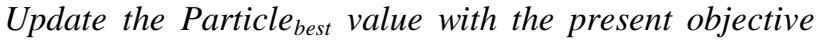
value.

END

END

Choose the particle with the best objective value of all as $G_{\text {best }}$.

FOR each particle

Estimate the velocity of the particle according to (5).

Update the position of the particles according to (6).

END

WHILE not reaching the maximum iterations or minimum error criteria.

RETURN $G_{\text {best }}$.

\subsection{Eagle Strategy (ES)}

Eagle strategy is a two phase scheme, created by Yang, and Deb [6]. ES is propelled from the searching conduct of eagles that are flying arbitrarily in similarity to the Levy flights. It utilizes various artificial intelligent techniques that make local and global explorations for fitting diverse purposes.

\subsection{Proposed ES-PSO}

Since ES is a two stage scheme, it utilizes different metaheuristic techniques at different stages. In ES based metaheuristic techniques, the search process in optimization problem can utilize randomization by means of Levy flights. With regards to AI techniques, the so-called Levy distribution [6] is a continuous probability distribution of sum of $\mathrm{N}$ independently and identically non-negative random variable, whose property function is expressed in Fourier transform (FT) as [6]-[9],

$$
F_{N}(k)=e^{-N|k|^{\beta}}
$$

The inverse transform to obtain the Levy distribution $\mathrm{L}(\mathrm{s})$ is not straight forward, the integral not having analytical form as,

$$
L(s)=\frac{1}{\pi} \int_{0}^{\infty} \cos (\tau s) e^{-\alpha \tau^{\beta}} d \tau \quad(0<\beta \leq 2)
$$

Here $\mathrm{L}(\mathrm{s})$ is called the Lévy distribution with an index $\beta$.

For the subsequent stage, PSO technique is as the intensifier local exploration. PSO is a global search technique; it can be optimized without much of a stretch to do productive local exploration by constraining new feasible solutions around a new local search areas.

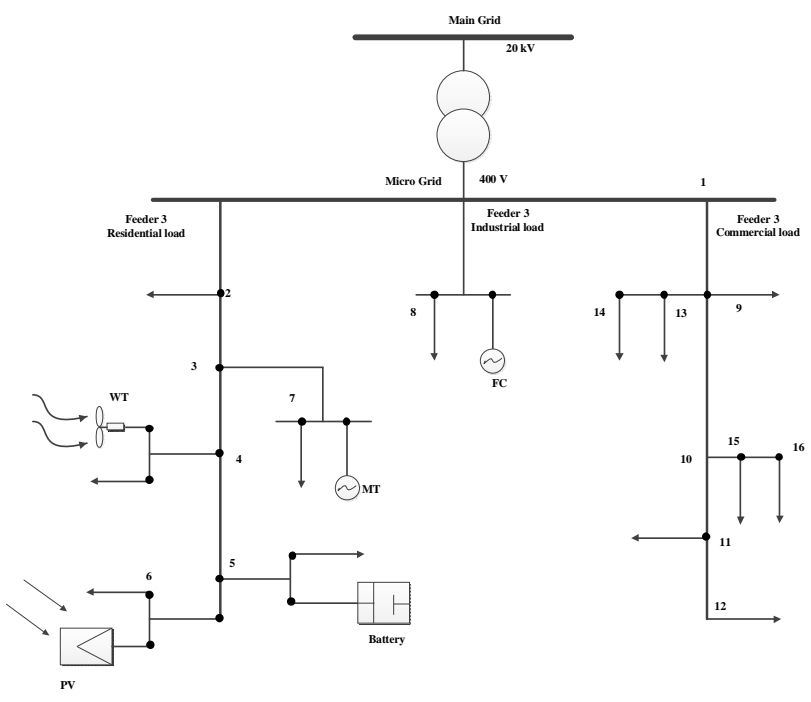

Figure 1: Low voltage micro grid

\section{RESULT AND DISCUSSIONS}

In this paper, the presented ES-PSO technique is employed to minimize the total operating cost of a low voltage micro grid as depicted in Figure 1 [3]. Furthermore, the problem is resolved in two different scenarios such as grid connected mode and islanded operation in order to gain better perceptive on the proposed ES-PSO technique.

\subsection{Simulation Data}

The forecasted market energy price for 24 hours is shown in Table 1 [3]. For scheduling of optimal set points to all DGs for both grid connected and islanded cases, all RESs are assumed to be operated for whole 24 hours i.e., status of all DGs are 1, therefore there is no start-down or shut-up cost for all DGs in these cases. The bids and limits of the DGs, BES and Grid are depicted in Table 2. The predicted power output of WT and $\mathrm{PV}$ are detailed in Table 3. To check the quality of the presented hybrid ES-PSO method, the simulation outcomes are compared with the existing algorithms developed in [3]. 
K. Santhosh et al., International Journal of Emerging Trends in Engineering Research, 8(9), September 2020, $5104-5110$

Table 1: Forecasted market price

\begin{tabular}{cc}
\hline Hour & $\begin{array}{c}\text { Market price } \\
(\mathbf{f c t} / \mathbf{k W h})\end{array}$ \\
\hline 1 & 0.230 \\
2 & 0.190 \\
3 & 0.140 \\
4 & 0.120 \\
5 & 0.120 \\
6 & 0.200 \\
7 & 0.230 \\
8 & 0.380 \\
9 & 1.500 \\
10 & 4.000 \\
11 & 4.000 \\
12 & 4.000 \\
13 & 1.500 \\
14 & 4.000 \\
15 & 2.000 \\
16 & 1.950 \\
17 & 0.600 \\
18 & 0.410 \\
19 & 0.350 \\
20 & 0.430 \\
21 & 1.170 \\
22 & 0.540 \\
23 & 0.300 \\
24 & 0.260 \\
\hline
\end{tabular}

Table 2: The bids and limits of the DGs, BES and Grid

\begin{tabular}{cccccc} 
ID & Type & $\begin{array}{c}\text { Bid } \\
(\mathbf{f c t} / \mathbf{k W h})\end{array}$ & $\begin{array}{c}\text { Start-up / } \\
\text { Shut-down } \\
(\mathbf{f c t})\end{array}$ & $\begin{array}{c}\mathbf{P}_{\min } \\
(\mathbf{k W})\end{array}$ & $\begin{array}{c}\mathbf{P}_{\max } \\
(\mathbf{k W})\end{array}$ \\
\hline 1 & MT & 0.4570 & 0.96 & 6.00 & 30.00 \\
2 & FC & 0.2940 & 1.65 & 3.00 & 30.00 \\
3 & PV & 2.5840 & 0.00 & 0.00 & 25.00 \\
4 & WT & 1.0730 & 0.00 & 0.00 & 15.00 \\
5 & BES & 0.3800 & 0.00 & -30.00 & 30.00 \\
6 & Grid & - & - & -30.00 & 30.00 \\
\hline
\end{tabular}

\subsection{Grid Connected Mode}

In Table 4, all AI techniques such as GA, PSO, fuzzy self adaptive PSO (FSAPSO), adaptive modified PSO based on tent equation (AMPSO-T), adaptive modifies PSO based on logistic equation (AMPSO-L), chaotic PSO based on logistic equation (CPSO-L), chaotic PSO based on tent equation (CPSO-T), and the proposed ES-PSO are compared for 20 trails for the objective function shown in (1) [3]. For better comprehension of the ES-PSO execution, the convergence property of ES-PSO against the conventional PSO technique in minimizing the total operating cost of micro grid is depicted in Figure 2. Similarly, the optimal power generation of DGs, battery power and grid power using the proposed ES-PSO algorithm are tabulated in Table 5.
Table 3: Forecasted power output of WT and PV

\begin{tabular}{ccc}
\hline Hour & $\begin{array}{c}\text { PV output } \\
\text { power } \\
(\mathbf{k W})\end{array}$ & $\begin{array}{c}\text { WT output } \\
\text { power } \\
(\mathbf{k W})\end{array}$ \\
\hline 1 & 0.0000 & 1.7855 \\
2 & 0.0000 & 1.7855 \\
3 & 0.0000 & 1.7855 \\
4 & 0.0000 & 1.7855 \\
5 & 0.0000 & 1.7855 \\
6 & 0.0000 & 0.9142 \\
7 & 0.0000 & 1.7855 \\
8 & 0.1937 & 1.3017 \\
9 & 3.7540 & 1.7855 \\
10 & 7.5279 & 3.0854 \\
11 & 10.4412 & 8.7724 \\
12 & 11.9640 & 10.4133 \\
13 & 23.8934 & 3.9228 \\
14 & 21.0493 & 2.3766 \\
15 & 7.8647 & 1.7855 \\
16 & 4.2208 & 1.3017 \\
17 & 0.5389 & 1.7855 \\
18 & 0.0000 & 1.7855 \\
19 & 0.0000 & 1.3017 \\
20 & 0.0000 & 1.7855 \\
21 & 0.0000 & 1.3017 \\
22 & 0.0000 & 1.3017 \\
23 & 0.0000 & 0.9142 \\
24 & 0.0000 & 0.6124 \\
\hline
\end{tabular}

\subsection{Islanded Operation}

In islanded operation, the micro grid is isolated from the main utility grid and all DGs including MT, FC, PV, WT and battery are operate to meet power demands. The convergence property of ES-PSO against the conventional PSO technique in minimizing the total operating cost of micro grid in islanded operation is depicted in Figure 3. Similarly, the optimal power generation of DGs and battery power in islanded mode using the proposed ES-PSO algorithm are tabulated in Table 6 .

Table 4: Comparison results for 20 trials

\begin{tabular}{lcccc}
\hline Algorithm & $\begin{array}{c}\text { Worst } \\
\text { Solution } \\
\text { (fct/day) }\end{array}$ & $\begin{array}{c}\text { Average } \\
\text { (€ct/day) }\end{array}$ & $\begin{array}{c}\text { Best } \\
\text { Solution } \\
\text { (€ct/day) }\end{array}$ & SD \\
\hline GA & 304.5889 & 290.4321 & 277.7444 & 13.4421 \\
PSO & 303.3791 & 288.8761 & 277.3237 & 10.1821 \\
FSAPSO & 291.7562 & 280.6844 & 276.7867 & 8.3301 \\
CPSO-T & 286.5409 & 277.4045 & 275.0455 & 6.2341 \\
CPSO-L & 281.1187 & 276.3327 & 274.7438 & 5.9697 \\
AMPSO-T & 275.0905 & 274.9821 & 274.5507 & 0.321 \\
AMPSO-L & 274.7318 & 274.5643 & 274.4317 & 0.0921 \\
ES-PSO & $\mathbf{2 7 4 . 1 9 9 5}$ & $\mathbf{2 7 3 . 9 2 9 2}$ & $\mathbf{2 7 3 . 8 7 6 6}$ & $\mathbf{0 . 0 8 4}$ \\
\hline
\end{tabular}


K. Santhosh et al., International Journal of Emerging Trends in Engineering Research, 8(9), September 2020, $5104-5110$

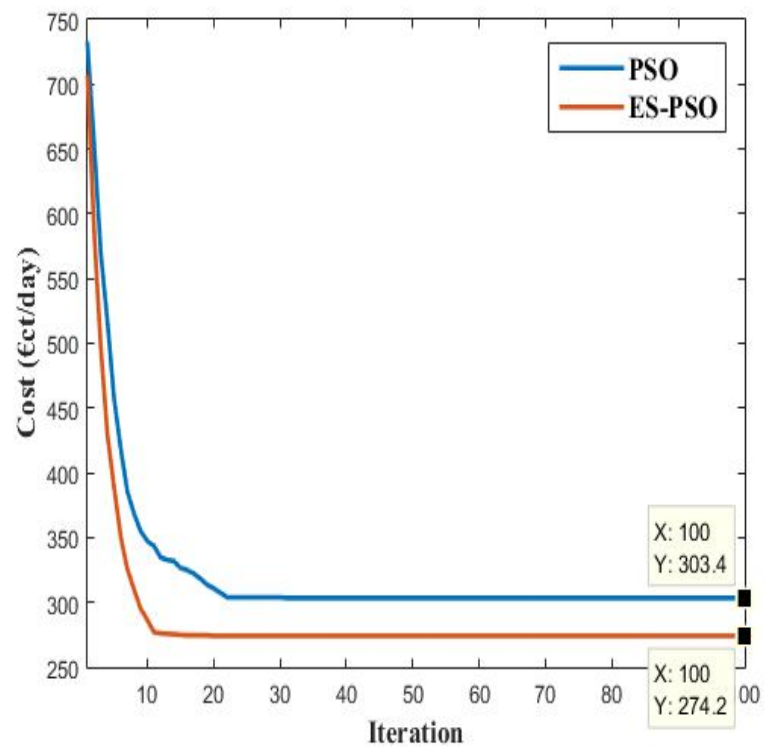

Figure 2: Convergence property - Grid connected mode

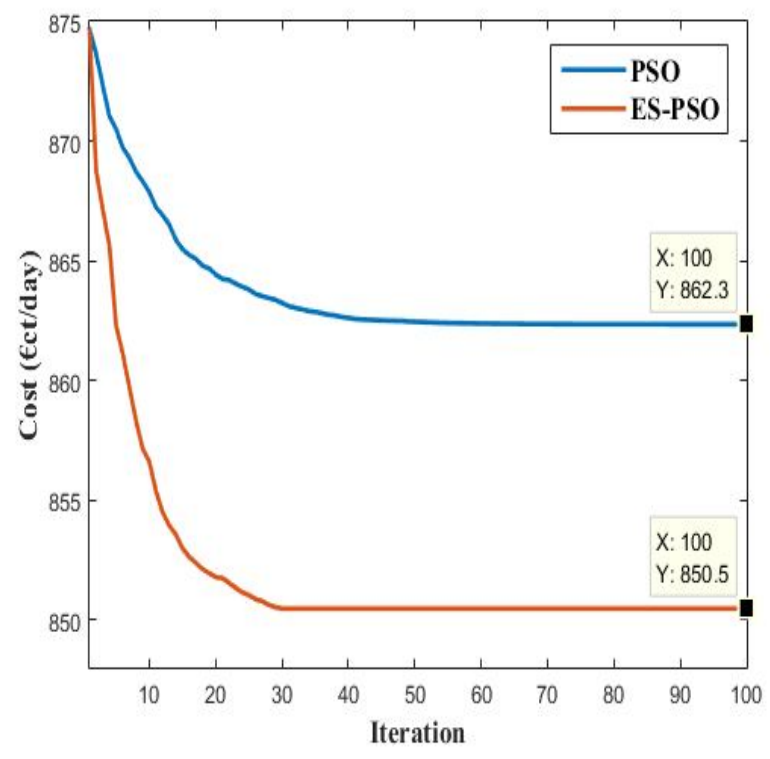

Figure 3: Convergence property - Islanded operation

Table 5: Optimal power output of DGs, battery and grid

\begin{tabular}{|c|c|c|c|c|c|c|c|}
\hline \multirow{2}{*}{$\begin{array}{c}\text { T } \\
(\mathbf{h})\end{array}$} & \multirow{2}{*}{$\begin{array}{c}\mathbf{P}_{\mathbf{L}} \\
(\mathbf{k W})\end{array}$} & \multicolumn{6}{|c|}{$\begin{array}{l}\text { DG units, Battery and Grid - Output Power } \\
\qquad(\mathrm{kW})\end{array}$} \\
\hline & & MT & FC & WT & PV & Battery & Grid \\
\hline 1 & 52.000 & 6.0000 & 30.000 & 1.7855 & 0.0000 & -15.785 & 30.0000 \\
\hline 2 & 50.000 & 6.0000 & 30.000 & 1.7855 & 0.0000 & -17.785 & 30.0000 \\
\hline 3 & 50.000 & 6.0000 & 30.000 & 1.7855 & 0.0000 & -17.785 & 30.0000 \\
\hline 4 & 51.000 & 10.653 & 30.000 & 1.7855 & 0.0000 & -21.439 & 30.0000 \\
\hline 5 & 56.000 & 6.0000 & 30.000 & 1.7855 & 0.0000 & -11.785 & 30.0000 \\
\hline 6 & 63.000 & 17.648 & 29.999 & 0.9142 & 0.0000 & -15.562 & 30.0000 \\
\hline 7 & 70.000 & 14.949 & 24.919 & 1.7855 & 0.0000 & -1.6540 & 30.0000 \\
\hline 8 & 75.000 & 6.0000 & 30.000 & 1.3017 & 0.1937 & 13.4549 & 24.0497 \\
\hline 9 & 76.000 & 30.000 & 30.000 & 1.7855 & 3.7540 & 30.0000 & -19.5395 \\
\hline 10 & 80.000 & 30.000 & 30.000 & 3.0854 & 7.5279 & 30.0000 & -20.6133 \\
\hline 11 & 78.000 & 28.786 & 30.000 & 8.7724 & 10.441 & 30.0000 & -30.0000 \\
\hline 12 & 74.000 & 21.622 & 30.000 & 10.413 & 11.964 & 30.0000 & -30.0000 \\
\hline 13 & 72.000 & 14.183 & 30.000 & 3.9228 & 23.893 & 30.0000 & -30.0000 \\
\hline 14 & 72.000 & 21.335 & 29.998 & 2.3766 & 21.049 & 27.2403 & -30.0000 \\
\hline 15 & 76.000 & 30.000 & 30.000 & 1.7855 & 7.8647 & 30.0000 & -23.6502 \\
\hline 16 & 80.000 & 30.000 & 30.000 & 1.3017 & 4.2208 & 30.0000 & -15.5225 \\
\hline 17 & 85.000 & 30.000 & 30.000 & 1.7855 & 0.5389 & 30.0000 & -7.3244 \\
\hline 18 & 88.000 & 12.706 & 30.000 & 1.7855 & 0.0000 & 30.0000 & 13.5079 \\
\hline 19 & 90.000 & 6.0000 & 30.000 & 1.3017 & 0.0000 & 22.6983 & 30.0000 \\
\hline 20 & 87.000 & 20.673 & 30.000 & 1.7855 & 0.0000 & 30.0000 & 4.5406 \\
\hline 21 & 78.000 & 30.000 & 30.000 & 1.3017 & 0.0000 & 30.0000 & -13.3017 \\
\hline 22 & 71.000 & 30.000 & 30.000 & 1.3017 & 0.0000 & 30.0000 & -20.3017 \\
\hline 23 & 65.000 & 6.0000 & 30.000 & 0.9142 & 0.0000 & -1.9143 & 30.0000 \\
\hline 24 & 56.000 & 16.869 & 30.000 & 0.6124 & 0.0000 & -21.481 & 30.0000 \\
\hline
\end{tabular}


K. Santhosh et al., International Journal of Emerging Trends in Engineering Research, 8(9), September 2020, $5104-5110$

Table 6: Optimal power output of DGs and battery

\begin{tabular}{|c|c|c|c|c|c|c|}
\hline \multirow{2}{*}{$\begin{array}{c}\text { T } \\
(\text { h) }\end{array}$} & \multirow{2}{*}{$\begin{array}{c}\text { PL } \\
(\mathbf{k W})\end{array}$} & \multicolumn{5}{|c|}{$\begin{array}{c}\text { DG units and Battery - Output Power } \\
(\mathrm{kW})\end{array}$} \\
\hline & & MT & FC & WT & PV & Battery \\
\hline 1 & 52.000 & 8.825 & 30.000 & 1.786 & 0.000 & 11.390 \\
\hline 2 & 50.000 & 6.000 & 30.000 & 1.786 & 0.000 & 12.215 \\
\hline 3 & 50.000 & 10.263 & 30.000 & 1.786 & 0.000 & 7.951 \\
\hline 4 & 51.000 & 6.000 & 30.000 & 1.786 & 0.000 & 13.214 \\
\hline 5 & 56.000 & 8.856 & 30.000 & 1.786 & 0.000 & 15.359 \\
\hline 6 & 63.000 & 6.148 & 30.000 & 0.914 & 0.000 & 25.938 \\
\hline 7 & 70.000 & 11.692 & 30.000 & 1.786 & 0.000 & 26.523 \\
\hline 8 & 75.000 & 16.061 & 30.000 & 1.302 & 0.194 & 27.443 \\
\hline 9 & 76.000 & 14.723 & 29.994 & 1.786 & 3.754 & 25.743 \\
\hline 10 & 80.000 & 22.295 & 29.999 & 3.085 & 7.528 & 17.093 \\
\hline 11 & 78.000 & 15.507 & 29.999 & 8.772 & 10.441 & 13.280 \\
\hline 12 & 74.000 & 18.088 & 30.000 & 10.413 & 11.964 & 3.535 \\
\hline 13 & 72.000 & 7.736 & 30.000 & 3.923 & 23.893 & 6.448 \\
\hline 14 & 72.000 & 10.812 & 30.000 & 2.377 & 21.049 & 7.762 \\
\hline 15 & 76.000 & 8.807 & 30.000 & 1.786 & 7.865 & 27.543 \\
\hline 16 & 80.000 & 18.355 & 29.997 & 1.302 & 4.221 & 26.126 \\
\hline 17 & 85.000 & 22.676 & 30.000 & 1.786 & 0.539 & 30.000 \\
\hline 18 & 88.000 & 26.215 & 30.000 & 1.786 & 0.000 & 30.000 \\
\hline 19 & 90.000 & 28.698 & 30.000 & 1.302 & 0.000 & 30.000 \\
\hline 20 & 87.000 & 25.215 & 30.000 & 1.786 & 0.000 & 30.000 \\
\hline 21 & 78.000 & 16.698 & 30.000 & 1.302 & 0.000 & 30.000 \\
\hline 22 & 71.000 & 15.815 & 30.000 & 1.302 & 0.000 & 23.883 \\
\hline 23 & 65.000 & 20.603 & 30.000 & 0.914 & 0.000 & 13.483 \\
\hline 24 & 56.000 & 6.000 & 30.000 & 0.612 & 0.000 & 19.388 \\
\hline
\end{tabular}

\section{CONCLUSION}

In this paper, hybrid metaheuristic approach with eagle strategy (ES) and particle swarm optimization (PSO) technique has been presented to minimize the operation cost of a low voltage micro grid with renewable energy source (RES) such as photovoltaic (PV), fuel cell (FC), micro turbine (MT), wind turbine (WT), and battery, while satisfying the load demand and system operating constraints. The presented hybrid approach has been analyzed on a typical micro grid and its better execution property has been compared with PSO, GA, AMSPO and CPSO. The simulation results indicated that the proposed hybrid ES-PSO technique has a superior performance in solving the micro grid optimization problems and also it has fast convergence properties when compared to other metaheuristic techniques.

\section{REFERENCES}

1. D.A. López-García, J.P. Torreglosa, and D. Vera. A decentralized P2P control scheme for trading accurate energy fragments in the power grid, International Journal of Electrical Power \& Energy Systems, Vol. 110, pp. 271-282, 2019.

2. M. Mansour-lakouraj, and M. Shahabi. Comprehensive analysis of risk-based energy management for dependent micro-grid under normal and emergency operations, Energy, Vol. 171, pp. 928-943, 2019.

3. A.A. Moghaddam, A. Seifi, T. Niknam, and M.R.A. Pahlavani,. Multi-objective operation management of a renewable MG (micro-grid) with back-up micro-turbine/fuel cell/battery hybrid power source, Energy, Vol. 36(11), pp. 6490-6507, 2011.

4. N. Bahmani-Firouzi, and R. Azizipanah-Abarghooee. Optimal sizing of battery energy storage for micro-grid operation management using a new improved bat algorithm, International Journal of 
K. Santhosh et al., International Journal of Emerging Trends in Engineering Research, 8(9), September 2020, $5104-5110$

Electrical Power \& Energy Systems, Vol. 56, pp. 42-54, 2014.

5. S. Sharma, S. Bhattacharjee, and A. Bhattacharya. Operation cost minimization of a micro-grid using quasi-oppositional swine influenza model based optimization with quarantine, Ain shams Engineering journal, Vol. 9(1), pp. 45-63, 2018

6. X.S. Yang, and S. Deb. Eagle strategy using Lévy walk and firefly algorithms for stochastic optimization, In Nature Inspired Cooperative Strategies for Optimization, Springer, Berlin, Heidelberg, 2010, pp. 101-111.

7. R. Eberhart, and J.Kennedy. Particle swarm optimization, In Proceedings of the IEEE international conference on neural networks, 1995, Vol. 4, pp. 1942-1948.

8. A.H. Gandomi, X.S. Yang, S. Talatahari, and S. Deb. Coupled eagle strategy and differential evolution for unconstrained and constrained global optimization, Computers \& Mathematics with Applications, Vol. 63(1), pp. 191-200, 2012.

9. K. Santhosh, and R. Neela. Optimal Placement of Distribution Generation in Micro-Grid using Eagle Strategy with Particle Swarm Optimizer, International Journal of Pure and Applied Mathematics, Vol. 118(18), pp. 3819-3825, 2018.

10. A.Satif, L. Hlou, H. Dahou, M. Mekhfioui, and R. Elgouri. Grid-connected photovoltaic systems synchronization algorithms under disturbances: a low-cost hardware implementation using Arduino DUE, International Journal of Emerging Trends in Engineering Research, 8(3), pp. 674-682, 2020

11. N. Nahak, O. Satapathy, S. Behera, M. Washiullah, and A. Naik. Optimal setting of governor parameters of a grid connected micro grid to damp power system oscillations, International Journal of Emerging Trends in Engineering Research, Vol. 8(6), pp. 674-682, 2020

12. B., Papari, C.S. Edrington, T.V. Vu, and F. Diaz-Franco. (2017, June). A heuristic method for optimal energy management of DC microgrid, In 2017 IEEE Second International Conference on DC Microgrids, IEEE, 2017, pp. 337-343. 